\title{
Editorial
}

\section{Cytokines, P38 MAP Kinase and the Pathophysiology of Depression}

\author{
\begin{tabular}{l} 
Andrew H Miller*,' and Charles L Raison' \\
'Department of Psychiatry and Behavioral Sciences, Emory University School of Medicine, Atlanta, GA, USA \\
\hline
\end{tabular} \\ Neuropsychopharmacology (2006) 3 I, 2089-2090. doi: I 0. I 038/sj.npp. I 301032
}

Increasing data suggest that innate immune inflammatory processes may contribute to behavioral changes that occur in the context of psychiatric disorders (Raison et al, in press). For example, in patients with major depression, all of the cardinal features of inflammation have been described, including elevations in proinflammatory cytokines, chemokines, cellular adhesion molecules, and acute phase proteins. In addition, administration of innate immune cytokines, most notably, interferon- $\alpha$ for the treatment of infectious diseases and cancer, has been found to lead to depressive syndromes in up to $50 \%$ of patients depending on the dose (Capuron and Miller, 2004). While much attention has been paid to immunologic contributions to behavioral comorbidities in the context of a wide range of medical disorders and their treatments, mounting data indicate that exposure to stress, a well-known precipitant of mood disorders, can also activate inflammatory responses both in the periphery and the brain. In addition, the mechanisms by which inflammatory responses are activated by stress including the role of sympathetic nervous system pathways have been described (Raison $e t$ al, in press).

Most relevant to the pathophysiology of mood disorders, research has identified an expanding list of overlapping pathways which are known to be involved in the development of depression and are recognized to be influenced by proinflammatory cytokines including monoamine neurotransmission, neuroendocrine function, and synaptic plasticity (Raison et al, in press). An excellent example of this convergence of relevant pathways involved in depression and influenced by cytokines is found in the current issue of Neuropsychopharmacology. In a manuscript by Zhu and co-workers, intriguing in vitro evidence is provided indicating another point of attack by which proinflammatory cytokines can influence the function of serotonergic pathways in the brain. Using a rat embryonic raphe cell line (RN46A) and mouse midbrain and striatal synaptosomes,

\footnotetext{
* Correspondence: Dr AH Miller, Department of Psychiatry and Behavioral Sciences, Emory University School of Medicine, 101 Woodruff Circle, Suite 4000, Atlanta, GA 30322, USA, Tel: + I 404 727 8260, Fax: + I 404727 3233, E-mail: amill02@emory.edu
}

these investigators have shown that treatment with either the proinflammatory cytokine IL-1 or TNF is associated with increased activity of the serotonin transporter; an effect mediated by activation of p38 mitogen-activated protein (MAP) kinase in IL-1-treated preparations. These data suggest that IL-1 or TNF in the brain (in addition to other relevant factors with the capacity to activate the MAP kinase signaling cascade) could contribute to decreased synaptic availability of serotonin. Interestingly in this regard, several studies have demonstrated that immune activation is also associated with decreased availability of tryptophan, the primary amino-acid precursor of serotonin (Capuron et al, 2002). This effect appears to be mediated in part through induction of the enzyme, indolamine 2,3, dioxygenase, which metabolizes tryptophan to kynurenine and ultimately quinolinic acid, which itself exhibits neurotoxic properties. The combination of increased serotonin reuptake coupled with decreased tryptophan availability may represent a double hit on serotonin neurotransmission, and shed light on findings that immune activation (as manifested by increased IL-6) in patients with major depression has been associated with resistance to treatment with the serotonin reuptake inhibitor, amitriptyline (Lanquillon et al, 2000). Moreover, reduction of depression-associated increases in TNF- $\alpha$ were a feature of amitriptyline responders (Lanquillon et al, 2000).

Activation of p38 MAP kinase by cytokines has also been shown to contribute to other pathologies relevant to depression. In several studies, p38 has been shown to disrupt transactivation of the glucocorticoid receptor, thus potentially contributing to glucocorticoid resistance (Wang et al, 2004). Glucocorticoid resistance is a primary feature of major depression as reflected by an abnormal dexamethasone (DEX) suppression test or DEX-corticotropin-releasing hormone test. In addition, p38 MAP kinase activation by bacterial endotoxin (lipopolysaccharide, LPS) has been shown to contribute to LPS-induced production of nitric oxide and expression of NO synthase (iNOS) mRNA in C6 glioma cells, an effect that can be inhibited by activation of protein kinase A (Won et al, 2001). Of note, increased NO and iNOS have been implicated in oxidative stress which has been shown to alter promoter function for several genes 
central to synaptic plasticity (Lu et al, 2004). Thus, cytokine activation of p38 appears to be an interesting common denominator contributing to several pathophysiologic pathways in major depression and thus may represent an interesting target for therapeutic development.

\section{REFERENCES}

Capuron L, Miller AH (2004). Cytokines and psychopathology: lessons from interferon-alpha. Biol Psychiatry 56: 819-824.

Capuron L, Ravaud A, Neveu PJ, Miller AH, Maes M, Dantzer R (2002). Association between decreased serum tryptophan concentrations and depressive symptoms in cancer patients undergoing cytokine therapy. Mol Psychiatry 7: 468-473.
Lanquillon S, Krieg JC, Bening-Abu-Shach U, Vedder H (2000). Cytokine production and treatment response in major depressive disorder. Neuropsychopharmacology 22: 370-379.

Lu T, Pan Y, Kao SY, Li C, Kohane I, Chan J et al (2004). Gene regulation and DNA damage in the ageing human brain. Nature 429: 883-891.

Raison CL, Capuron L, Miller AH Cytokines sing the blues: Inflammation and the pathogenesis of major depression. Trend Immunol (in press).

Wang X, Wu H, Miller AH (2004). Interleukin-1 alpha-induced activation of p38 mitogen-activated kinase inhibits glucocorticoid receptor function. Mol Psychiatry 9: 65-75.

Won JS, Lee JK, Suh HW (2001). Forskolin inhibits expression of inducible nitric oxide synthase mRNA via inhibiting the mitogen activated protein kinase in C6 cells. Molecular Brain Res 89: 1-10. 